\title{
Marco analítico para la gobernanza territorial. La política pública de infancia y adolescencia en Colombia*
}

\author{
Analytical Framework for Territorial Governance. \\ Public Policy on Childhood and Adolescence in Colombia \\ Marco analítico para governança territorial. A política \\ pública da infância e adolescência na Colômbia \\ OMAIRA ORDUZ R. ${ }^{* *}$ \\ Pontificia Universidad Javeriana (Bogotá, Colombia)
}

JAVIER A. PINEDA D. ${ }^{* * *}$

Universidad de los Andes (Bogotá, Colombia)

\footnotetext{
* Este artículo es producto de varios proyectos de investigación. En primer lugar, la investigación realizada por Omaira Orduz como trabajo de tesis en el marco de la Maestría en Política Social de la Pontificia Universidad Javeriana, titulada «Gobernanza de la política pública de infancia y adolescencia». En segundo lugar, un proyecto de investigación realizado por Javier Pineda en el Cider (Universidad de los Andes), sobre «Gobernanza y desarrollo local», parte del cual fue publicado en Pineda (2014). Artículo de investigación recibido el 23.11.2017 y aceptado el 21.02.2019.

** Email: orduzomaira@hotmail.com

*** Email: jpineda@uniandes.edu.co ORCID: http://orcid.org/oooo-ooo3-1183-7677
} 


\section{Cómo citar}

ORduZ, OMAIRA y PinedA, JAVIER A. (2019). Marco analítico para la gobernanza territorial. La política pública de infancia y adolescencia en Colombia. Revista CS, 27, 89-116. https://doi.org/10.18046/recs.i25.2588 
Resumen

Abstract

Resumo

El presente artículo, con base en una revisión del concepto de gobernanza, examina la política pública de infancia y adolescencia a nivel local en Colombia. El examen empírico de la gobernanza se realiza a partir de las categorías analíticas de dirección y liderazgo, la interacción de actores, espacios de decisión y estructura multinivel, el diseño y la estructura institucional, la capacidad técnica de los niveles de gobierno y la rendición de cuentas. Estas categorías muestran los múltiples aspectos que inciden en la configuración de la gobernanza local de esta política y ayudan a entender por qué se logra o limita la garantía de los derechos prevalentes de la niñez en los territorios a partir de dos estudios de caso.

PALABRAS CLAVE:

gobernanza, política pública, infancia y adolescencia, desarrollo local, Colombia

This article, based on a review of the concept of governance, examines the public policy of childhood and adolescence at local level in Colombia. The empirical examination of governance is carried out based on the analytical categories of management and leadership, the interaction of actors, decision spaces and multilevel structure, the design and institutional structure, the technical capacity of the levels of government and accountability. These categories show the multiple aspects that affect the configuration of local governance of this policy and understand why the guarantee of the prevailing rights of children in the territories is achieved or limited, based on two case studies.

\section{KEYWORDS:}

Governance, Public Policy, Childhood and Adolescence, Local Development, Colombia 
Este artigo, baseado em uma revisão do conceito de governança, avalia a política pública da infância em nível local na Colômbia. O exame empírico da governança realiza-se com base nas categorias analíticas de liderança, a interação de atores, espaços de decisão e estrutura multinível, o desenho e a estrutura institucional, a capacidade técnica dos níveis de governo e a rendição de contas. Essas categorias mostram os múltiplos aspectos que afetam a configuração da governança local desta política e entender por que a garantia dos direitos vigentes das crianças nos territórios é alcançada ou limitada a partir de dois estudos de caso.

PALAVRAS-CHAVE:

governança, políticas públicas, infância e adolescência, desenvolvimento local, Colômbia 


\section{Introducción}

Desde la declaración de las Metas de Desarrollo del Milenio, en la cumbre de Naciones Unidas de 2000, se reconoció la importancia central de la gobernanza, y su vínculo con el desarrollo y los derechos humanos. Después de cerca de dos décadas de recorrido en la búsqueda de las metas del desarrollo, se reconoce que la gobernanza debe jugar un papel más fuerte en los Objetivos de Desarrollo Sostenible (ODS), para lograr instituciones y sistemas efectivos de gobernanza que sean transparentes y responsables en brindar servicios esenciales a las necesidades públicas, y promover el crecimiento inclusivo (PNUD, 2014).

En la literatura académica, la gobernanza se ha consolidado en las últimas dos décadas como un concepto válido para el análisis de las políticas públicas y el desarrollo desde distintos enfoques institucionalistas. En este campo, el análisis de la gobernanza local ha tomado relevancia. Carmona (2004) establece que el territorio es el lugar de construcción social y política, y es una instancia organizadora del desarrollo, en la cual una diversidad de actores públicos, privados, institucionales y sociales desarrollan su acción colectiva. El desarrollo local lo vincula al proceso político de la gobernanza, en el cual el actor estatal promueve articulaciones más cooperativas y participativas en las que fluyen múltiples intereses locales y relaciones de poder que despliegan sus capacidades de negociación para la toma de decisiones.

La gobernanza en el desarrollo local es también analizada por Brito (2002), quien concluye que el ámbito local es donde se construyen los espacios para la concertación ciudadana entre los diferentes sectores sociales, económicos y políticos, pues integra su cultura. Frente a la pregunta de cómo se conjuga la gobernanza en el nivel local y las políticas públicas, Aguilar (2010) establece que la política pública «representa el punto donde se plasma y es visible la gobernanza» (50) y es precisamente en este campo en el que con mayor «frecuencia y visibilidad» (51) los gobiernos ponen en evidencia las limitaciones institucionales, políticas, técnicas y directivas.

Jolly (2002) considera el territorio como un lugar de construcción política. Las dinámicas de cercanía permiten que confluyan la elaboración e implementación de las políticas públicas (policies) con el juego político (politics). Este autor establece el vínculo entre la descentralización como proyecto político y las políticas públicas generadas por la misma descentralización. Identifica, además, que en la descentralización en Colombia operan dos lógicas: una sectorial (reglamenta el territorio) y otra territorial (gobiernan los territorios), con lo cual fundamenta su hipótesis de políticas públicas que combinan centralismo y descentralización (Jolly, 2002: 8).

Con base en una revisión de literatura sobre gobernanza, el presente artículo identifica distintos usos y dimensiones del concepto, a partir de lo cual avanza en 
la propuesta de un marco analítico para el estudio de la gobernanza local. Para esto se tuvo en cuenta el marco analítico de la gobernanza realizado por Hufty (2011b), pero se propusieron unas categorías analíticas de un nivel teórico más concreto y específico para el análisis de la gobernanza local de la política en examen. Así, se construyó una propuesta metodológica de investigación, a partir de cinco categorías analíticas que permiten la conexión entre la teoría y la realidad de la gobernanza, estas son: la dirección y el liderazgo; la interacción de actores, espacios de decisión y estructura multinivel; el diseño y la estructura institucional; la capacidad técnica de los niveles de gobierno; y la rendición de cuentas o accountability local. Con este marco analítico, se abordó la gobernanza local o municipal de la política de infancia y adolescencia en Colombia.

Si bien a nivel nacional e internacional se vienen adelantando estudios sobre gobernanza en diferentes áreas y campos de las ciencias sociales, son escasos los estudios de esta materia a nivel municipal en políticas sociales. El presente artículo surge de una investigación cualitativa que se desarrolla a partir de estudios de caso a nivel municipal, con el objetivo de explicar la manera como se configura la gobernanza de la Política Pública de Infancia y Adolescencia en Colombia (PPIA). El propósito de la investigación es responder las siguientes preguntas: ¿cómo se ha configurado la gobernanza de la PPIA en el nivel municipal? y ¿cuáles han sido las principales dificultades para la territorialización de esta política en el ejercicio de la gobernanza local?

Para esto, fueron seleccionados dos municipios del departamento de Boyacá: Aquitania y Sogamoso. Este departamento es, después de Antioquia, el que más número de municipios tiene en Colombia (123), lo cual permitía mostrar patrones más generales de operación en lo local. El municipio de Sogamoso se seleccionó porque su «Plan de Desarrollo 'Sogamoso Piensa en Grande' (2008-2011)» fue calificado, por el Departamento Nacional de Planeación (DNP), como el segundo en el país en haber incluido la población de niños, niñas y adolescentes, en respuesta a la expedición del «Código de Infancia y Adolescencia» en 2006. Internacionalmente, fue presentado como experiencia exitosa, por lo cual se quiso conocer este caso. El municipio de Aquitania fue seleccionado como un municipio pequeño (categoría seis), de vocación rural, que tipifica la mayoría de los municipios del departamento.

La recolección de información se realizó mediante entrevistas semiestructuradas a los actores estratégicos de la gobernanza local, como alcaldes, jefes de planeación, funcionarios nacionales, departamentales y municipales del sistema nacional de bienestar, lideresas comunitarias, etc. En algunos casos, se realizó observación participante en instancias de coordinación de la política. 


\section{El concepto de gobernanza y marcos analíticos para su estudio}

El concepto de gobernanza tiene una evolución teórica muy reciente, $\mathrm{y}$ aun cuando el término viene del francés antiguo (gouvernance), su uso frecuente y proliferación en la literatura anglosajona bajo el término de governance se desarrolló principalmente en la década de los noventa. Este concepto atrajo un gran interés y múltiples traducciones e interpretaciones, en la medida en que busca explicar la forma como funcionan los gobiernos, su eficacia en el cumplimiento de sus funciones y el desarrollo de las políticas públicas, la influencia del marco institucional, y la participación e interacción de los actores de acuerdo con los diferentes roles, responsabilidad e intereses. Su esencia reside en concretar o materializar los objetivos del Estado a través de una acción pública cada vez más compleja, diversa y plural en contextos de descentralización y globalización económica, social y cultural (Cerrillo, 2005; Madrueño-Aguilar, 2017).

Los organismos multilaterales para el desarrollo jugaron un papel importante en este proceso. El uso del término governance se expandió a partir de 1989 con el Informe del Banco Mundial en el que se sostenía que la incapacidad de los gobiernos africanos para gobernar y administrar era la causa de su falta de desarrollo sostenible. El informe del PNUD (1997), «Reconceptualizing Governance», vincula el concepto con los procesos de desarrollo, recopila la producción teórica a la fecha y define la gobernanza como «el conjunto de mecanismos, procesos, relaciones e instituciones mediante las cuales los ciudadanos y grupos articulan sus intereses, ejercen sus derechos y obligaciones, y concilian sus diferencias» (10). Este informe identifica como instancias claves al Estado, las organizaciones de la sociedad civil y el sector privado, y establece que «el sistema de gobernanza es la estructura institucional formal y organizativa del proceso de decisiones vinculantes del Estado moderno» (PNUD, 1997:31). Aunque estos enfoques han sido criticados por los contextos políticos de condicionalidad que imponen las agencias bilaterales y multilaterales de desarrollo, han sido claramente normativos en la promoción de modelos específicos de gobernanza, y evaden el análisis de las relaciones asimétricas del poder que se presentan en ella (Hufty, 2011a).

Hufty (2011a), en el marco de un amplio programa de investigación, revisa lo que considera son los enfoques más populares del concepto: gobernanza corporativa, gobernanza global, buena gobernanza y gobernanza moderna. Su principal objetivo es desarrollar un enfoque para el concepto y un método para usarlo, para lo cual desarrolló dos fases: una primera exploratoria, que consistió en la revisión de los enfoques existentes, y otra que fue el desarrollo de un marco teórico y metodológico 
de la gobernanza. Para este último, publicó un texto separado (Hufty, 2011b), en el cual desarrolla el marco analítico de la gobernanza (Governance Analytical Framework, GAF) como un instrumento para sistematizar la complejidad social, política e institucional de los procesos de formulación e implementación de decisiones colectivas. Es decir, el GAF constituye una metodología para observar y analizar los procesos de gobernanza en cualquier sociedad. Para estos efectos, el autor brinda la siguiente definición del término:

La gobernanza hace referencia a una categoría de hechos sociales, esto es a los procesos de interacción y toma de decisión entre los actores involucrados en un problema colectivo que conlleva a una creación, reforzamiento o reproducción de normas sociales e instituciones. (Hufty, 2011b: 405).

Para esto, establece seis criterios que esta metodología debe cumplir: el GAF debe ser realista (no normativo), interdisciplinario, reflexivo, comparativo, generalizable y operacional. El primero de estos criterios resulta interesante debido a que el autor sustenta que los enfoques de gobernanza que revisa en su primer texto (Hufty, 2011a) son básicamente normativos, es decir, que promueven un tipo de gobernanza. En tal sentido, señala que el GAF debe optar por una postura epistemológica que promueva una descripción y análisis realista de la gobernanza. No obstante, opta por un construccionismo suave o un realismo crítico, donde la descripción y el análisis deben estar lo más cerca de lo no normativo y no prescriptivo, mientras la interpretación puede estar basada en enfoques subjetivos.

Con base en lo anterior, Hufty define el GAF sobre la base de cinco herramientas analíticas coherentemente vinculadas; estas son: problemas, normas sociales, actores, puntos nodales y procesos. El autor soporta teóricamente cada una de estas categorías, especialmente en el legado sociológico de Pierre Bourdieu. Sin duda, esta es la propuesta de marco analítico más elaborada en la literatura, soportada por varios estudios empíricos donde ha sido utilizada. No obstante, el carácter universalista o generalizable de este marco analítico que -en criterio del autorpuede aplicarse a sociedades inclusive sin Estado parte de un problema colectivo, socialmente construido, que puede o no ser objeto de política pública. Este marco analítico, sin embargo, en cuanto se afianza en el institucionalismo sociológico, se encuentra muy cerca de las propuestas conceptuales para el análisis de política pública, como el enfoque de las coaliciones defensoras de Sabatier (1999), entre otros enfoques (Pineda, 2018).

Teniendo en cuenta las categorías analíticas de Hufty, se revisaron algunos autores, con el fin de obtener unas categorías analíticas de un nivel teórico más concreto y específico para el análisis de la gobernanza territorial en políticas públicas. Esto 
no pretende revisar el GAF universal propuesto por Hufty, sino abrir el abanico de instrumentos metodológicos para el análisis de gobernanza territorial. La revisión de literatura en esta investigación permitió, por un lado, apreciar elementos metodológicos útiles entre los principales autores y, por otro, diferentes énfasis en la definición y uso del concepto. De acuerdo con los enfoques que revisa Hufty (2011a), se analizaron algunos autores que vale la pena reseñar.

Desde el enfoque de la gobernanza moderna, Rhodes (1996), autor escocés, señala que la gobernanza se refiere a la autoorganización y a las redes interorganizacionales, las cuales complementan los mercados y las jerarquías de gobierno, destinan recursos de manera confiable, y ejercen control y coordinación. Rhodes analiza distintos usos populares, pero imprecisos, del término. Dentro de las más relevantes se encuentra la noción desde la Nueva Administración Pública (NAP), para la cual la gobernanza puede ser vista como la dirección (que introduce los conceptos de gestión del sector privado al sector público) y como la Nueva Economía Institucional (que introduce estructuras de incentivos de mercado) en la provisión de servicios públicos. Mientras que la una se encamina al desempeño por resultados de gestión, la otra se preocupa por desagregar burocracias y elecciones de consumo, entre otros elementos (Rhodes, 1996: 655).

Después de realizar una crítica a estos enfoques, y a partir del análisis del caso del gobierno británico, Rhodes determina que el elemento clave en la definición del concepto es su contribución al análisis de los cambios en el gobierno en contextos de privatización, pérdida de las funciones del gobierno central y local, y limitaciones de los servidores públicos. La visión intraorganizacional de la NAP deja de lado el manejo interorganizacional e intergubernamental. En este análisis, se encuentran tres características distintivas que son: la solución de problemas, los juegos intergubernamentales y la conexión entre redes. ${ }^{1}$ Como conclusión, considera que la gobernanza (definida como una red interorganizacional autoorganizada) ayuda a entender el cambio en los gobiernos debido a que, primero, identifica el área sobre la cual las redes proveen servicios y solución a los problemas que resultan de la conducción y la transparencia; segundo, muestra los límites que tienen las reformas desde la NAP, las cuales se preocupan por el control intraorganizacional y el manejo por objetivos o competencias, enmascarando la necesidad de la confianza y la cooperación con

1. En Rhodes (1996), cuando se hace referencia al enfoque de gobernanza como redes de autoorganización, se utiliza el término red para describir la interdependencia entre los diversos actores en la provisión de servicios. A su vez, las redes son creadas por organizaciones que necesitan intercambiar recursos para lograr sus objetivos, maximizar la influencia sobre sus resultados y evitar llegar a ser dependientes de otros actores del proceso (6;8). 
el lenguaje del mercado; y, por último, sugiere que las redes requieren un estilo de manejo basado en la facilitación, adaptación y el pacto.

El trabajo pionero de Jan Kooiman (1993), Modern Governance: New Government-Society Interactions, de la Universidad de Rotterdam, Holanda, distingue los conceptos de «gobernar» del gobierno, y el de «gobernanza» como un proceso nuevo de dirección de la sociedad, esta última caracterizada por relaciones dinámicas e interdependientes con el gobierno y de este con el sector privado. El autor explica cómo existe una capacidad limitada de la administración pública, en la medida en que «ningún actor, público o privado, tiene la información y el conocimiento requerido para resolver problemas y situaciones 'complejos, dinámicos y diversificados', ni tiene el mirador suficiente para seleccionar los instrumentos eficaces y menos aún el poder suficiente para dominar unilateralmente» (251). Además, establece cómo las pautas tradicionales de gobernar que eran de «tráfico en un solo sentido», del gobernante a los gobernados, se han modificado para dar cabida a un «tráfico en dos sentidos», que nutre el modo de gobernar en doble vía.

Posteriormente, Kooiman (2004) identifica y destaca algunos elementos comunes de la diversidad de definiciones del término gobernanza, así: «las reglas y las cualidades de los sistemas, la cooperación para incrementar la legitimidad y la eficiencia y la atención a nuevos procesos y acuerdos público-privados» (171). Aunque incluye las reglas, su énfasis continúa siendo la interacción y cooperación de los gobernantes y los privados, con líneas divisorias e intereses que son cada vez más difusos, y fija como argumento central de la gobernanza moderna la «combinación de todo tipo de actividades y estructuras de gobierno» (172) que denomina como modos y órdenes. Otros autores, como Brito (2002), Carmona (2004), Jolly (2002), Pineda (2014) y Ruano (2002), se ubican en este énfasis de la gobernanza. En cuanto todos ellos se inspiran en análisis institucionalistas, aportan elementos a las categorías de análisis propuestas por Hufty de la interacción de los actores, los puntos nodales y los procesos de gobernanza.

Otro énfasis que define la gobernanza como reglas de juego ha tenido una amplia aceptación. Uno de los autores más influyentes, el catalán Joan Prats (2003), considera la gobernanza como una herramienta analítica de «procesos de interacción de los actores estratégicos» (244), cuyos patrones de relacionamiento e interacción «tienen su fundamento último en las reglas del juego que restringen el comportamiento de los actores que cumplen una doble función: 1. Solucionar dilemas distributivos (Bardham, 1999) y 2. Solucionar problemas de información (Shepsle y Weingast, 1994)» (244). Prats (2003) establece que los conceptos de gobernabilidad y gobernanza no son excluyentes, sino, por el contrario, complementarios, para lo cual se apoya en 
las distinciones que hacen Kooiman (1993) y otros autores, y concluye que la gobernabilidad es la capacidad conferida por la interacción de los actores estratégicos (gobernanza) que produce la arquitectura institucional y que permite reforzar al mismo sistema sociopolítico para transformar necesidades ciudadanas en políticas.

En un trabajo posterior, Prats (2005) analiza cómo la globalización transforma las prácticas de la política democrática de los estados y señala que hay un ensanchamiento del poder de los medios de comunicación, lo cual exige de la gestión pública mayores capacidades de liderazgo, trabajo en equipo, comunicación, concertación y gestión para resolver los conflictos entre los diferentes actores de los diversos niveles del gobierno (147). Así, otorga un papel central a los procesos de dirección y liderazgo en el análisis de la gobernanza; aspecto que también es tenido en cuenta por Aguilar (2010) en su texto Gobernanza: el nuevo proceso de gobernar.

Este concepto de gobernanza con énfasis en las reglas del juego está, sin duda, anclado al institucionalismo económico, puesto que utiliza una definición muy semejante a la que North (1993) otorga para las instituciones. Alejandro Portes (2006), desde el legado histórico de la sociología, realiza una crítica al institucionalismo económico y define las instituciones diferenciando las categorías de valores, normas, roles e instituciones, en el campo de la cultura y en su relación con el campo del poder, de acuerdo con su nivel causal de influencia y de visibilidad en la realidad. Este enfoque desde el institucionalismo sociológico es muy coherente con el utilizado por Hufty. Así, de acuerdo con esta perspectiva, la categoría de normas sociales de Hufty (2011b) se sitúa en un nivel intermedio de abstracción, mientras la categoría de instituciones -como formas de interacción consuetudinaria de los actores, según Portes- se encuentra en un nivel más concreto. Por tal razón, se utilizará aquí la categoría de instituciones como interacción de actores.

Para avanzar desde el concepto amplio de gobernanza dado por Hufty hacia una gobernanza territorial, se hace también necesario precisar lo que se conoce como gobernanza multinivel.

\section{Gobernanza multinivel}

Este es un aspecto transversal de la gobernanza que especifica que la interacción de los actores estratégicos se produce en diferentes niveles del gobierno que requieren coordinación y articulación. Charbit (2011) establece que los gobiernos subnacionales juegan un papel central en la formulación e implementación de las políticas públicas y en la efectividad de las mismas, lo cual depende no solo de la interdependencia que exista, sino también de las responsabilidades y funciones que estos actores 
cumplan, y considera la gobernanza multinivel como necesaria para la gestión de las políticas públicas.

El enfoque de gobernanza multinivel, (multi-level governance, MLG) que Charbit (2011) entiende como «el intercambio implícito y explícito de la autoridad en la formulación de políticas, la responsabilidad, el desarrollo y la aplicación en los diferentes niveles administrativos y territoriales» (13), se circunscribe a una interacción de poderes públicos de distintos actores de las capas de gobierno del orden local, regional, provincial, nacional y supranacional, es decir, en «las relaciones intergubernamentales». No obstante, este concepto restringido a los niveles de gobierno, que excluye los demás actores no públicos, será ampliado para su uso en el presente estudio. ${ }^{2}$

Del mismo modo, Ruano (2002) señala que la política pública «adopta la forma de gobernanza a múltiples niveles» (1), lo que implica que no depende de un actor individualmente considerado, sino que, por el contrario, depende de los contextos del entorno socio organizativo de la acción pública, que incluyen los problemas, la toma de decisiones y su ejecución. Prats (2005) ratifica la importancia de la interacción de actores, en diferentes niveles, en la definición que hace de gobernanza como «el modo de gobernación característico de una sociedad compleja, diversa, interdependiente y dinámica en la que las administraciones actúan con racionalidad limitada, el conocimiento se halla fragmentado entre diversos actores y la realización de los intereses generales depende de la calidad de la interacción entre las administraciones públicas, lo que podemos definir como gobierno multinivel, y de éstas con las organizaciones de la sociedad civil y del sector privado» (13).

Finalmente, existen diferentes análisis del papel del Estado en la gobernanza. Los autores anglosajones Peters (1980, como se citó en Cerrillo, 2005) y Pierre (2000, como se citó en Cerrillo, 2005) consideran que el Estado es un actor relevante y tiene un papel preponderante en la dirección, en su condición de centro del poder político. Carmona (2004), igualmente, considera que en la gobernanza la centralidad la tiene el Estado. Hay otros autores que analizan la pérdida del rol protagónico del Estado, como es el caso de Rhodes (1996), Kooiman (2004) y Le Galès (1998), que consideran a este como un actor más dentro del proceso político. Por otra parte, la autora Renate Mayntz (2005), socióloga alemana, establece cómo el Estado se reserva el derecho a la ratificación legal y la autorregulación de la sociedad, pero

2. La gobernanza multinivel, según este autor, requiere, para la gestión de las políticas públicas, contextos de descentralización, independientemente de si estos son óptimos o no. Charbit (2011) diferencia, de acuerdo con su naturaleza, las clases de interdependencia entre los niveles de gobierno, en: institucional, financiera y socioeconómica, contextos en los cuales las responsabilidades y los resultados están completamente relacionados. 
bajo principios u ordenadores diferentes «a la sombra de la jerarquía» (89), para conservar en la gobernanza un papel estratégico.

Este nuevo papel del Estado, en los diferentes niveles, a través de la gobernanza, lleva a la pregunta de la legitimidad del sistema democrático dentro de las limitaciones y posibilidades que se dan en la realidad política. Jolly (2002) considera el territorio como un lugar de construcción política, y Cerrillo (2005), compilador del libro Gobernanza hoy, señala que las relaciones entre la gobernanza y la democracia deben ser complementarias. Por su parte, Carmona (2004) establece que el territorio es también el lugar de construcción social y política, y agrega que es una instancia organizadora del desarrollo en la cual una diversidad de actores públicos, privados, institucionales y sociales desarrollan su acción colectiva.

\section{Marco analítico para la gobernanza territorial}

De acuerdo con el análisis teórico realizado, se identificaron los siguientes elementos de la gobernanza para el análisis de la formulación e implementación de la PPIA en los casos de estudio. Estos elementos centrales se constituyen en categorías analíticas que permiten vincular la teoría con la realidad y conocer componentes concretos para el análisis de la gobernanza. Aunque no se pretende establecer un marco universal único, y mucho menos acabado, se considera que estas categorías pueden ser útiles para el análisis de la gobernanza territorial en otros campos de la política pública distinto al abordado en este caso.

1. Proceso de dirección y liderazgo: se trata de analizar los actores en el proceso político y social de tomar iniciativas que definen objetivos colectivos, las acciones para conseguirlos y la forma de coordinar y organizar dichas acciones (Aguilar, 2010; Prats, 2005). Se busca conocer cómo operan las habilidades de los gobernantes y otros actores para fortalecer los procesos con las comunidades, identificar las necesidades, leer el contexto social y económico, orientar la gestión con políticas pertinentes, movilizar recursos y negociar las condiciones de desarrollo con otros niveles de gobierno (Brito, 2002: 263). La interacción de múltiples actores gubernamentales, privados, organizaciones de la sociedad civil y ciudadanía se presenta mediante instancias y espacios de decisión para facilitar la cooperación y coordinación; no obstante, el peso e influencia, tanto de la sociedad como del gobierno, es cambiante. «Se resalta el proceso por encima del actor y el proceso social por encima del actor gubernamental» (Aguilar, 2010: 37).

2. Interacción de actores, espacios de decisión y estructura multinivel: esta determina el tipo de actores, nivel en que se coordina, relaciones entre los diferentes niveles, patrones de interacción entre los actores estratégicos y, ante todo, espacios de decisión 
en contextos específicos que permitan transformar los problemas en políticas (Prats, 2003: 445), y la calidad de la interacción (Prat, 2005: 130). Esta interacción humana está guiada por un sistema de creencias basadas en percepciones de los actores, bajo las cuales entienden los problemas y adoptan decisiones con el propósito de reducir la incertidumbre de las organizaciones políticas, sociales y económicas (Roth, 2003). Aquí se unifican dos categorías del GAF de Hufty (2011b): las normas sociales y los puntos nodales. Portes (2006) distingue las instituciones de los valores, las normas y los roles, y precisa su relación con las estructuras sociales de poder. Retomando el legado sociológico, este autor señala que «las instituciones gobiernan las relaciones entre los ocupantes de roles en organizaciones sociales» (Portes, 2006: 241), por lo cual, en el nivel de influencia causal, las instituciones no solo se diferencian de las normas sociales, sino que son más visibles que aquellas. Las formas consuetudinarias de interacción de los actores o las instituciones son así una categoría analítica más concreta que las normas sociales. Por su parte, los espacios de interacción y decisión corresponden a los puntos nodales del modelo de Hufty (2011b).

3. Diseño y estructura institucional: la interacción entre los actores estratégicos se da también de acuerdo con las organizaciones involucradas, es decir, la arquitectura o arreglo institucional existente en los distintos niveles territoriales. Esto afecta el carácter de las políticas públicas y, a su vez, estas establecen reglas de juego o marcos institucionales que regulan la toma de otras decisiones colectivas. En este elemento, las relaciones de poder se derivan del sistema político, el sistema electoral, la forma de gobierno -ya sea dividido o unificado-, las condiciones socioeconómicas y la ideología que afecta el sesgo de la política y la clase de política que se pretende implementar. «Las instituciones políticas son, en su sentido más básico, las reglas que rigen el juego político y sus interacciones con otros sistemas, como el social o el económico» (Prats, 2003: 246). En esta categoría se capta la estructura institucional que se genera en el campo específico de la gobernanza, entendido este bajo el concepto de Bourdieu (Bourdieu y Wacquant, 1995). Igualmente, desde el análisis de política pública, específicamente el enfoque de las coaliciones defensoras, es la configuración del subsistema de política, así denominado por Sabatier (1999).

4. Capacidad técnica de los niveles de gobierno: también llamada, por Aguilar (2010), dimensión técnico gerencial de la gobernanza, que representa «el conjunto de las condiciones técnicas, operativas y gerenciales que hacen que la actividad directiva que produce las preferencias sociales sea eficaz (...)» (38) en la formulación e implementación de la política pública. Allí mismo, se establecen reglas de juego para la interacción en la orientación de la vida social, y se siguen las normas legales y técnicas que rigen el comportamiento directivo (Aguilar, 2010: 44). En este proceso, la toma de decisiones se fundamenta «en información, conocimiento experto, saber tecnológico y competencias gerenciales específicas (...) con la estimación y asignación de los recursos (...), con la determinación de los compromisos (...), con la distribución de la autoridad y del 
trabajo, con las actividades de coordinación, seguimiento y evaluación de las actividades, productos y resultados» (Aguilar, 2010:39). Para la «territorialización» y municipalización de la política pública que se traduce en la formulación e implementación de la política, esta capacidad técnica está referida no solo a la identificación de los problemas públicos, la concreción de objetivos, la toma de decisiones y la puesta en marcha de acciones colectivas para su cumplimiento, sino también a los actores participantes, e instancias e instrumentos que se adopten para su desarrollo en cada nivel territorial.

5. Rendición de cuentas (accountability): Hernández (2013) señala que la rendición de cuentas es el «conjunto de modalidades de control, contrapeso y supervisión que los gobernantes, funcionarios y agentes privados utilizan en la gestión de recursos públicos» (5). Establece cómo la rendición de cuentas se constituye, desde la perspectiva relacional, en la forma de encuentro y articulación de múltiples actores, en tanto se relacionan quienes tienen la obligación con quienes tienen el derecho a exigir las cuentas.

La información, comunicación y transparencia en el manejo de la gestión y de los recursos públicos en los diferentes niveles de gobierno son elementos sustanciales de la gobernanza, apoyados hoy por las tecnologías de la información, a través de la cual se construye confianza con la comunidad que representa, se conoce el uso del patrimonio colectivo, permite hacer balance y ajuste, aporta ideas que pueden contribuir en la gestión, y fortalece los principios democráticos de transparencia y participación (Jolly, 2002: 18). Esta es quizás la única categoría analítica de tipo normativo en términos de la discusión que Hufty (2011a) sostiene con distintos enfoques de gobernanza, dada la importancia de la rendición de cuentas para la democracia.

Con estas categorías analíticas, se definió una estrategia de investigación basada en el estudio de caso, el cual ha sido considerado en la investigación sobre la gobernanza de las políticas públicas por su relevancia para ilustrar buenas prácticas, análisis de procesos de política y evaluación de programas.

\section{Territorialización de la política pública de infancia y adolescencia}

La Convención Internacional de los Derechos del Niño de 1989 es considerada como la Carta Magna de derechos humanos de la infancia y la adolescencia, y es tomada como la base jurídica para la refundación de una nueva ciudadanía cuyo sujeto de derecho son los niños y niñas en su plenitud, superando el concepto de «menores», término con el que se denominaba la incapacidad política de la infancia y la adolescencia en el enfoque de la situación irregular (García, 1998: 9-25). 
Con el contexto de América Latina, el Estado colombiano desarrolló también una legislación y unas políticas públicas que se pueden considerar robustas para la garantía de los derechos de todos los niños, niñas y adolescentes (en adelante NNA), y ha incorporado en el orden jurídico instrumentos internacionales de protección de los NNA, de acuerdo con lo dispuesto en el Artículo 93 de la Constitución Política de 1991 (y Artículos 44 y 45). De acuerdo con estos preceptos constitucionales, quince años después se produjo la Ley 1098 de 2006, conocida como el Código de Infancia y Adolescencia.

Del mismo modo, Colombia cuenta con una sólida formulación de política con planes nacionales, programas y proyectos en diferentes problemáticas públicas de la infancia y adolescencia, así: Política Pública de la Primera Infancia, ${ }^{3}$ Plan Nacional de Alimentación y Nutrición, ${ }^{4}$ política de prevención del trabajo infantil, política de atención al adolescente en conflicto con la ley penals (también llamado Sistema de Responsabilidad Penal para Adolescentes, SRPA), política de prevención al reclutamiento, ${ }^{6}$ estrategia de prevención del embarazo adolescente y, más recientemente, la política de Estado para el Desarrollo Integral de la Primera Infancia de Cero a Siempre. ${ }^{7}$

La Ley 1098 de 2006, en su Artículo 204, establece la responsabilidad indelegable en el diseño, ejecución y evaluación de las políticas públicas de infancia y adolescencia en los niveles nacional, departamental, distrital y municipales; en el presidente, los gobernadores y los alcaldes, a quienes se les exige además la realización de rendición de cuentas al respecto. La transferencia de competencias a los entes territoriales debe contar con permanentes mecanismos de acompañamiento para garantizar que, en todas las regiones, sin discriminación alguna, se ejerza, por parte de los niños y niñas, el goce de sus derechos.

Con este marco de política pública, los gobernantes de los distintos niveles deben incorporar y concretar en los planes de desarrollo, tanto en el orden nacional y departamental como municipal, la puesta en marcha de programas, proyectos y acciones que se soportan en los principios de protección integral, ${ }^{8}$ interés superior

3. Conpes Social 109 de 2007, Ley 12 de 2009, Decreto 4875 de 2011 y Ley 1804 de 2016.

4. Conpes 113 de 2008.

5. Conpes 3629 de 2009.

6. Conpes 3673 de julio de 2010 y Decreto 4690 de 2007, entre otros.

7. Ley 1804 de 2016.

8. Art. 7 de la Ley 1098 de 2006. 
de la niñez ${ }^{9}$ y prevalencia de sus derechos. ${ }^{10}$ Es importante anotar que la legislación nacional prevé como ente articulador de las entidades responsables de la política de infancia y adolescencia de los niveles nacional, departamental, distrital y municipal, al Sistema Nacional de Bienestar Familiar, cuyo ente rector es el Instituto Colombiano de Bienestar Familiar (ICBF) ${ }^{11}$

Esta investigación buscó conocer de qué manera se configuraba la gobernanza de la PPIA en los municipios del departamento de Boyacá (con 123), para lo cual seleccionó un municipio mediano (Sogamoso, categoría tres) y uno pequeño (Aquitania, categoría seis), en cumplimiento de las responsabilidades que les compete. ${ }^{12}$

El municipio de Aquitania, a una altura entre 3050 y 3500 metros sobre el nivel del mar, tenía 17 mil habitantes para 2015, de los cuales el 60 \% se encontraban en el área rural. Persisten los problemas relacionados de violencia intrafamiliar, embarazos no deseados en adolescentes, maltrato infantil y alto consumo de cerveza. No obstante contar con importantes fuentes naturales hídricas, la cobertura de agua potable en las zonas rurales del municipio es mínima. Sogamoso, a una altitud cercana a los 2600 metros sobre el nivel del mar, contaba con 117 mil habitantes en 2015, con un marcado predominio urbano $(85 \%)$.

Estos municipios comparten una tradición cultural de trabajo de niños y niñas en tareas propias de la agricultura y la minería. Los patrones culturales de relaciones patriarcales que caracterizan las relaciones políticas, familiares y sociales influyen directamente en las relaciones de trato con niños y niñas. Estos factores culturales se traducen en un débil reconocimiento de ellos y ellas como sujetos, lo que dificulta el ejercicio de sus derechos y la persistente exclusión de su participación.

\section{La gobernanza de la política de infancia y adolescencia en los municipios}

De acuerdo con las categorías analíticas definidas en la metodología, en esta sección se presentarán los resultados de investigación en los estudios de caso. Por efectos de extensión del artículo, no se incluirán muchos testimonios y evidencias del trabajo de campo que amplían y sustentan los argumentos, los cuales se sintetizan en esta sección.

\footnotetext{
9. Art. 8 de la Ley 1098 de 2006.

10. Art. 9 de la Ley 1098 de 2006.

11. Art. 205 de la Ley 1098 de 2006.

12. Art. 204 del Código de Infancia y Adolescencia.
} 


\section{Dirección y liderazgo}

Las alcaldías municipales, como responsables de la administración local, tienen un papel fundamental en la territorialización de la Política Pública de Infancia y Adolescencia (PPIA). Para el análisis del proceso de dirección y liderazgo político, se revisa en los municipios la forma como el gobernante -como actor estratégicoorienta, moviliza recursos, negocia, organiza y desarrolla la gestión municipal, para alcanzar los objetivos colectivos propuestos en el Plan de Desarrollo en este campo de la política pública.

En Aquitania, la dirección y liderazgo de la política de infancia y adolescencia es muy débil por parte del gobernante, en la medida en que, primero, se delega en funcionarias de poco poder a cargo de los temas de niñez y familia como un asunto de mujer, dándose un proceso de feminización de la política; segundo, desde la cultura política, se presenta una estrategia de respuesta formal ante las exigencias de los otros niveles departamentales y nacionales de gobierno; $y$, tercero, existe poco desarrollo de habilidades para orientar la gestión a fin de dar respuesta a las necesidades de la comunidad.

En cuanto al primer argumento, de acuerdo con la entrevista al alcalde en ejercicio, este señala que delega sus funciones en la comisaría de familia, y expresa falta de conocimiento y liderazgo al frente de la PPIA en el municipio. Lo anterior es corroborado por la misma comisaria cuando, al preguntársele sobre quiénes están asumiendo la responsabilidad de esta política en los municipios de la región, informa que en la mayoría de los casos son las comisarías de familia. Son ellas quienes, igualmente, tienen a cargo la Secretaría Técnica del Consejo de Política Social, instancia encargada de la dirección y coordinación de la política a nivel municipal.

Existe lo que se podría llamar una naturalización de la responsabilidad de la política en manos de las funcionarias de familia y sus equipos, en cuanto existe en el imaginario institucional que todo lo que tiene que ver con la niñez y la adolescencia está directamente relacionado con la gestión de la comisaria y las medidas de restablecimiento de derechos que estos funcionarios imponen. La niñez y la adolescencia sigue viéndose como un asunto de una de las dependencias de la administración municipal y en cabeza de mujeres, que son las que «cuidan» de los derechos, dejando de lado el principio de Protección Integral.

En cuanto al segundo argumento que sustenta la debilidad en la dirección y el liderazgo, se evidencia que, ante las exigencias del Sistema Nacional de Bienestar Familiar (SNBF) de contar con una política pública específica y diferencial de infancia y adolescencia que cumpla con los objetivos de orientar la acción y los recursos del Estado en la localidad, el alcalde expresa un desconocimiento del proceso al respecto, lo cual conlleva a la contratación de una consultoría para la realización 
del documento de política pública y, de esa manera, cumplir con el requisito formal, buscando lograr la legitimidad con el cumplimiento normativo, sin liderar el proceso y sus resultados.

Finalmente, el tercer elemento de debilidad identificado para la dirección y liderazgo está relacionado con las habilidades para orientar la gestión a fin de dar respuesta a las necesidades de la comunidad. Al respecto, no se identificaron procesos y habilidades de liderazgo que le permitan al Alcalde escuchar, direccionar y propiciar, no solo la comunicación con la ciudadanía, sino la articulación del equipo de la administración para responder con los temas que más preocupan a las comunidades en este campo de la política.

En Sogamoso se encuentra que la dirección y liderazgo son fuertes, en la medida que, primero, el gobernante moviliza y orienta recursos del Estado y la sociedad civil; segundo, asume directamente las funciones que le corresponden en la política pública, y brinda respuestas reales a los problemas y a las exigencias de la política con objetivos comunes; $\mathrm{y}$, tercero, ha desarrollado relativos conocimientos y habilidades para orientar la gestión a fin de dar respuesta a las necesidades de la comunidad.

El municipio de Sogamoso fue uno de los primeros municipios en el país en contar con una PPIA (desde 2010), luego de un proceso de gobernanza de dicha política en cabeza, directamente, del alcalde, que le valió reconocimiento nacional e internacional, en tanto que centró su administración alrededor de la garantía integral de los derechos de los NNA. Este hecho constituye un importante antecedente que determina la trayectoria de la política pública y su gobernanza en el presente.

El gobernante sucesor para el período 2012-2015 mantuvo claro su papel de dirección y la transversalidad de la política en su administración. El alcalde movilizó recursos, coordinó acciones, conoció las necesidades de los NNA e implementó estrategias para organizar la gestión municipal alrededor de la integralidad de sus derechos. Además, señaló que «trabajamos en diferentes líneas, buscamos ONGs que nos apoyen en el tema, aprovechamos las ayudas de las iglesias para llegar a las familias de los colegios, porque es como entrar a rescatar el tema en los núcleos familiares (...)».

En cuanto al segundo argumento que sustenta la fortaleza en la dirección y el liderazgo, con respecto a brindar respuestas reales a los problemas y a las exigencias de la política, el gobierno local cuenta con una política claramente formulada, que se hereda de la administración anterior. Este punto de quiebre que establece el gobierno predecesor genera una trayectoria institucional que se autosostiene (Mahoney, 2000). El «Plan de Desarrollo 'Sogamoso Ciudad Competitiva' 2012-2015», aprobado mediante Acuerdo Municipal N. ${ }^{\circ} 013$ de 2012, contempló el enfoque de desarrollo integral que guió la administración para el mencionado cuatrienio (Concejo Muni- 
cipal de Sogamoso, 2012: 8). Este Plan se realizó con consulta ciudadana mediante diferentes encuentros, y su capítulo segundo contiene un completo diagnóstico de la infancia y la adolescencia por categoría de derechos, con los correspondientes objetivos de la política y responsables en cada uno de ellos.

El tercer argumento sobre la dirección y el liderazgo en la gobernanza de la política en el municipio tiene que ver con el desarrollo de relativos conocimientos y habilidades que han logrado el alcalde y su equipo, para orientar la gestión a fin de dar respuesta a las necesidades de la comunidad. En los problemas de drogadicción, deserción educativa secundaria y media, embarazo adolescente, etc., se identificaron capacidades en el gobernante para hacer seguimiento e implementar ajustes en la gestión municipal relacionada con infancia y adolescencia.

\section{Interacción de actores, espacios y estructura multinivel}

En Aquitania, la interacción de los actores en los espacios de participación generados por la PPIA se caracteriza por un nivel bajo de contribución, articulación y eficacia, y por una relación multinivel, donde las entidades nacionales imponen ciertos requisitos y programas que bloquean la respuesta colectiva de los actores frente a las necesidades locales. Esta característica de la interacción, con la debilidad en la dirección y el liderazgo ya vista, conduce a que el municipio carezca no solo de una política pública específica para su territorio, sino también de proyectos propios, quedando a merced de programas nacionales sobre los cuales no ejerce ninguna autonomía. Así, la alta vulneración de los derechos de la infancia y la adolescencia de Aquitania no logra ser revertida ni por la dinámica local, ni por la territorialización de los programas nacionales.

El principal espacio de interacción de actores para la PPIA es el Consejo de Política Social (CPS) del municipio. Frente a la forma como se desarrolla este Consejo, la comisaria de familia, en su condición de Secretaria Técnica, dice: «Bueno, cumplimos con el reglamento nacional, que es por lo menos cumplir con las cuatro reuniones en el año». Aquí también se repite el cumplimiento formal de la norma, pero sin una gestión orientada a resultados.

En observación participante de la tercera sesión del $\mathrm{CPS}^{13}$ durante cuatro horas, se observó poca participación y una débil interacción entre los actores, en la medida

13. La tercera sesión del Consejo de Política Social de Aquitania se desarrolló el 10 de julio de 2013, al cual fueron convocadas 38 personas y llegaron 18 , en su mayoría funcionarios de la administración municipal. No hubo presencia de las 16 veredas que componen el municipio. La funcionaria departamental del ICBF señaló: «falta mayor participación de la comunidad (...) son voces que no son escuchadas, no hay participación (...)». 
que se socializaba de manera fragmentada la información de cada dependencia, sin que esta fuera objeto de discusión y articulación de esfuerzos que fortalecieran los procesos de la gestión pública. Es una reunión plana, donde el alcalde modera, los actores de los diferentes sectores exponen, los otros escuchan y quedan las respectivas actas. El ejercicio que se desarrolla en el CPS no corresponde al que se establece y pretende dentro de la PPIA, dado que no confluyen el Comité de primera infancia, las redes de buen trato, el programa de erradicación del trabajo infantil ni los planes para la erradicación de la explotación sexual comercial, entre otros.

A pesar de la difícil situación de los niños y niñas en el municipio de Aquitania, este no cuenta con proyectos específicos que atiendan dichas vulneraciones. Básicamente, los programas y proyectos que se ejecutan bajo los lineamientos que se señalan desde el nivel nacional son los de Familias en Acción, Red Unidos, Cero a Siempre, Desayunos con Amor (DIA), Programa de Alimentación Escolar (PAE), entre otros. Es decir que estos son proyectos que no llegan a las veredas y sobre los cuales el municipio no cuenta con ninguna autonomía para adecuarlos o ajustarlos a la situación del nivel local. Así, las graves particularidades de vulneración de los derechos de la infancia y la adolescencia en el municipio no son atendidas ni por la dinámica local, ni por los programas nacionales.

Con respecto a Sogamoso, las características de la interacción de los actores están relacionadas con los procesos adelantados por los dos gobiernos anteriores. El proceso de planeación participativa de la política abrió la deliberación e interacción de los distintos actores y movilizó a estos con la comunidad. La interacción que se identifica es horizontal, dinámica y con un proceso pedagógico con la comunidad en la búsqueda de una mayor comprensión y priorización de los derechos de la infancia y la adolescencia.

Por ejemplo, la política de madres gestantes logró involucrar a actores de la sociedad civil y de la empresa privada mediante un ejercicio de negociación, y la concertación para el transporte gratuito de mujeres embarazadas. En el proceso de coordinación y articulación de actores, se logró también movilizar recursos y apoyos técnicos de organizaciones del orden internacional, como es el caso de UNICEF y otras entidades. Según los indicadores, el proceso tuvo resultados concretos a corto plazo, pero sobre todo generó nuevas reglas de juego y una trayectoria que sus sucesores y contradictores políticos, en su mayoría, tuvieron que continuar, con algunas fracturas, en el desarrollo posterior de la política en el municipio, entre 2008 y 2015. A pesar de las fracturas de origen político relacionadas con los cambios en la administración, una regla de juego que no logró alterarse fue, justamente, la participación de la sociedad civil en instancias de coordinación, como fue el Consejo de Política Social del municipio, que garantizó, en buena medida, la continuidad de la política. 


\section{Diseño y estructura institucional}

Este elemento de la gobernanza se refiere a que las interacciones entre los actores estratégicos se desarrollan de acuerdo con la configuración o arquitectura institucional existente, lo cual afecta directamente a las políticas públicas. Este marco institucional regula la toma de decisiones y está permeado por las relaciones de poder que se derivan del sistema político y electoral, así como de la forma de gobierno, entre otros aspectos.

Para el caso de Aquitania, en entrevistas realizadas con lideresas comunitarias de las 16 veredas que componen el municipio, se recogieron amplios testimonios de cómo los derechos de niñas, niños y adolescentes no han sido garantizados por las administraciones municipales. Estas lideresas comentan la exclusión de que han sido objeto en las distintas administraciones por no hacer parte del grupo político respectivo. Las reglas de juego establecidas en la institucionalidad política hacen que primen unos patrones de interacción que dejan sin reconocimiento y espacios a sectores políticos que cuentan con conocimiento de la realidad que viven los niños y niñas en las veredas.

La institucionalidad política basada en una norma informal de gobernar sin buscar la mayor inclusión política genera un déficit de confianza y desperdicia recursos para el desarrollo de la política pública y del municipio. Se desconocen las capacidades, experiencias y el tejido social que se ha construido en el municipio con las familias, perdiendo con ello las posibilidades de transformaciones a través de políticas públicas pertinentes. Del mismo modo, el hecho de contratar un consultor para el diseño del documento de PPIA en el municipio, para cumplir con la exigencia del nivel nacional, redujo la interacción con actores estratégicos responsables. El interés político del gobernante local es cumplir con los requisitos formales ante los entes departamentales y nacionales, atender su clientela más urbana y los compromisos con los políticos regionales. Las reglas de juego formal brindan espacio para imponer unas informales dadas por los intereses de los actores políticos regionales en el poder.

En el caso de Sogamoso, el proceso de gobernanza de la PPIA determinó nuevas normas para la toma de decisiones colectivas. La institucionalidad gubernamental se transformó técnicamente, mientras el reconocimiento nacional del municipio y los logros alcanzados comprometieron a las siguientes administraciones, a pesar del cambio abrupto de gobernante y de partido político en la dirección de la ciudad. Con respecto a esto último, fue decisiva la participación de miembros del Concejo municipal -órgano de representación política local-, quienes en la formulación del Plan de Desarrollo 2012-2015 exigieron que se incluyera un capítulo sobre infancia y adolescencia exclusivamente, so pena de no ser aprobado. La negociación y 
concertación del plan entre el concejo y el alcalde llevaron también a fortalecer la institucionalidad y gobernanza de la PPIA, con la inclusión de organizaciones de la sociedad civil en las instancias de gestión de la política.

\section{Capacidad técnica de los distintos niveles de gobierno}

La capacidad técnica está relacionada con las condiciones técnicas, gerenciales y operativas que permiten que la actividad directiva y la toma de decisiones del gobernante sean eficaces. Para ello, adquiere un papel central la información, el conocimiento experto, el saber tecnológico, la consecución y asignación de recursos, la coordinación de las tareas, y el seguimiento y evaluación de las actividades. La capacidad técnica también está referida a la asistencia que deben recibir los municipios por parte de las instancias del orden tanto departamental como nacional, para que se realice de manera eficiente y efectiva la territorialización y municipalización de la política pública.

En relación con la asesoría técnica que debe brindar el departamento a los municipios en la formulación e implementación de la PPIA, no existe claridad institucional sobre en cabeza de quién se encuentra dicha responsabilidad al interior de la gobernación. ${ }^{14}$ Como lo informaron funcionarios departamentales, la tarea, de manera simultánea y limitada, la están desarrollando tanto la Secretaría de Desarrollo Humano como la de Planeación Departamental, lo cual evidenció falta de coordinación para la implementación de la política al interior de la entidad.

Por su parte, la Dirección Regional del ICBF, a quien le corresponde coordinar los doce centros zonales distribuidos en el departamento, ${ }^{15}$ cuenta con dos personas responsables de acompañar a los municipios en calidad de referentes del Sistema Nacional de Bienestar Familiar (SNBF), quienes intentan coordinar y articular la asistencia técnica de los doce centros en los 123 municipios. Una de ellas informa que la meta en Boyacá es acompañar técnicamente a 85 municipios que fueron los que firmaron el convenio que tiene como objeto aunar esfuerzos y recursos para articular las entidades de la política social y los comités de infancia y adolescencia. $\mathrm{Al}$ respecto, señala que «un primer momento fue convocar a los municipios para que firmen los convenios, eso nos tomó un año, [y en un segundo momento] hemos hecho un proceso de firmar actas de inicio (...), la idea es fortalecer la protección integral». Frente a la pregunta de por qué se suscribe convenio con los municipios,

14. El departamento de Boyacá cuenta con 123 municipios organizados en 13 provincias.

15. El ICBF cuenta con 33 regionales y 206 centros zonales en todo el país. 
la funcionaria responde que «desde la sede nacional, de acuerdo a cómo se maneja este proceso, nos dieron la orden».

Los anteriores apartes permiten, claramente, señalar, como se evidenció en el municipio de Aquitania, que el SNBF, a través de reglas formales de carácter vertical, pretende, mediante un proceso administrativo con los municipios, dar cumplimiento a las exigencias de la Ley 1098 de 2006, relacionadas con que cada uno de ellos cuente con una PPIA diferencial, sin que realmente se desarrollen estrategias de acompañamiento técnico y soluciones directas a las problemáticas en sus territorios. Por su parte, los gobernantes locales se preocupan por cumplir con la entrega formal de un documento de PPIA diferencial.

El SNBF logró la suscripción de 999 convenios en 27 gobernaciones, pero la asesoría técnica se desarrolla en un estilo top-down, desde «arriba», y se aplica a los de «abajo», lo que significa un desconocimiento e invisibilidad de las propuestas del territorio y una relación de poder vertical a través de la cual se complacen requerimientos políticos sin que se analice, entre muchos otros elementos, la capacidad de respuesta de los equipos municipales y el mismo recurso humano para adelantar dicha tarea.

A julio de 2013, solo 20 municipios - de 123 del departamento- contaban con PPIA. El SNBF fue creado en 1979 y, no obstante, en materia de implementación de política cuenta con una débil capacidad técnica, un centralismo administrativo y técnico, y una gestión guiada por requerimientos formales, mas no por resultados. La complementariedad y subsidiaridad de la nación en la territorialización y municipalización de la política termina para muchos en un cumplimiento formal más que deben cumplir los alcaldes para no ser sancionados por los órganos de control.

\section{Rendición de cuentas o accountability local}

Este es un elemento que consagró el Artículo 204 de la Ley 1098 de 2006, en el cual al gobernante territorial, como responsable indelegable de la PPIA, le corresponde la obligación de rendir cuentas. Este es un proceso relacional entre los actores que tienen el deber con quienes tienen el derecho a exigir los resultados de la gestión pública y del manejo de los recursos públicos (Hernández, 2013).

Es importante resaltar que, no obstante, la exigencia de la Estrategia Hechos y Derechos liderada por la Procuraduría General de la Nación con otras entidades, para que los gobernantes rindan cuentas frente al tema de infancia y adolescencia, usualmente se incluye como un aparte en la rendición de cuentas general que los gobiernos locales realizan. Según testimonio de los funcionarios locales y departamentales, en ningún municipio se rinden cuentas de manera específica para la PPIA, dada la poca articulación de la sociedad civil que exija espacios específicos para ello. 


\section{Conclusiones}

La gobernanza no solo es una elaboración conceptual de origen relativamente reciente y, por lo tanto, en construcción, sino que también presenta distintas dimensiones que resulta preciso analizar para su mejor comprensión. En tal sentido, un primer elemento de conclusión consiste en señalar las bondades y los límites que el marco conceptual definido presentó para el análisis de la gobernanza de la política de infancia en Colombia.

La mayoría de los autores ha coincidido en que la gobernanza surge para explicar el nuevo contexto de redefinición del papel de los gobiernos y la importancia de la acción conjunta de los diversos actores en la sociedad. Con esto, se busca explicar los estilos de gobierno en los cuales las fronteras entre lo público y lo privado no son claras, centrados en los mecanismos de gobierno que no descansan en las clásicas ideas de la autoridad del Estado, sino en la interacción e interdependencia de múltiples actores (Aguilar, 2007; Brito, 2002; Kooiman, 2004). El análisis de la política de infancia y adolescencia en los municipios colombianos permitió observar que, si bien es a partir de los arreglos institucionales existentes que la dirección y el liderazgo los pueden ejercer los gobernantes de turno, la acción de actores diferentes al Estado, como las lideresas comunitarias o las organizaciones de la sociedad civil, resultan centrales para la trayectoria institucional y la gobernanza de la política. Así, la sociedad civil es decisiva en la garantía de los derechos de la infancia y la adolescencia en municipios medianos y pequeños, pues actúa a través de redes con miembros de las corporaciones públicas (caso Sogamoso) o a través de lideresas comunitarias (caso Aquitania).

Resultó también muy útil el concepto de gobernanza que pone el énfasis en explicar la forma como funcionan los gobiernos, su eficacia en el cumplimiento de sus funciones y el desarrollo de las políticas públicas, la influencia del marco institucional y la participación e interacción de los actores de acuerdo con los diferentes roles, responsabilidad e intereses, en contextos de descentralización y globalización. En tal sentido, la forma consuetudinaria de interacción de los actores, es decir, las instituciones formales e informales que establecen el comportamiento de los actores, fue central (Prats, 2003), al igual que los elementos de gobernanza multinivel (Charbit, 2011) y los aportes de la gobernanza al análisis de las políticas públicas (Jolly, 2002).

No obstante, dos limitaciones que la literatura aún no ha desarrollado con mayor claridad, que no fueron abordadas en este trabajo y que surgen como un elemento para otros proyectos de investigación, consisten en, primero, la relación entre el sistema político y la gobernanza de la política pública y, segundo, las reglas de juego más informales, relacionadas justamente con el funcionamiento del sistema político 
y la cultura local. Si bien algunos autores mencionan estos temas (Aguilar, 2010; Jolly, 2002), son escasos los trabajos específicos sobre la materia.

Un segundo elemento de conclusiones está relacionado con hallazgos generales de la investigación. En primer lugar, a pesar de que el Sistema Nacional de Bienestar Familiar cuenta con un andamiaje institucional construido durante más de tres décadas, la política de infancia y adolescencia no cuenta con una clara gestión territorial, debido, en buena medida, a factores relacionados con la gobernanza que se evidenciaron en el presente estudio. La falta de claridad de objetivos, la ausencia de un diseño institucional para la gestión de la política, la prevalencia de prácticas formales y dispersas para adelantar una asistencia técnica fluida y articulada con los gobiernos locales, y la carencia de una interacción horizontal y consensuada del quehacer de la política, son elementos que afectan la gobernanza de la política y la atención de las particularidades de los NNA en los municipios.

El territorio, entendido como espacios de construcción colectiva (Jolly, 2002), posibilita que la administración y organización local puedan solucionar las problemáticas de la cotidianidad que afrontan los niños, niñas y adolescentes en sus contextos, a través de la formulación e implementación de las políticas públicas y del juego político. No obstante, esta cercanía con las comunidades, por si sola, no necesariamente produce articulaciones más consensuadas de los actores locales que logren como resultado transformaciones en la garantía y protección de los derechos de los NNA.

En segundo lugar, una de las distintas formas de concebir la infancia y la adolescencia está relacionada directamente con la protección y el cuidado que las mujeres, históricamente, les han brindado. Hoy, dicha concepción aún se ve reflejada en patrones de la gestión de la política pública en estudio, en la medida que se delega la responsabilidad del tema a las comisarías de familia en la mayoría de municipios. En los municipios, igualmente, se encontró que los secretarios de planeación, a pesar de ser una de las políticas bajo su responsabilidad, no se quieren ver comprometidos, puesto que feminizan la política porque de ese tema «dan cuenta las mujeres».

\section{Referencias}

AGUILAR, L. (2007). El aporte de la política pública y la Nueva Gestión Pública a la gobernanza. XII Congreso Internacional sobre la Reforma del Estado y la Administración Pública, Santo Domingo.

AGUILAR, L. (2010). Gobernanza: El nuevo proceso de gobernar. Ciudad de México: Friedrich Naumann Stiftung für die Freiheit. 
BOURDIEU, P. y WACQUANT, L. (1995). Respuestas. Por una antropología reflexiva. México: Grijalbo.

BRITO, M. (2002). «Buen gobierno» local y calidad de la democracia. Instituciones y Desarrollo, $12-13,249-275$.

CARMONA, R. (2004). El desarrollo local como desafio político. Nuevas formas de gobierno y gestión participativa en el territorio. Recuperado de http://www.ag.org.ar/3congreso/Ponencias/Carmona.pdf

CERRILLO, A. (2005). La gobernanza hoy: 10 textos de referencia. Madrid: Instituto Nacional de Administración Pública.

CHARBIT, C. (2011). Governance of Public Policies in Decentralised Contexts: The Multi-level Approach, OECD Regional Development Working Papers, 2011/o4. http://dx.doi.org/10.1787/5kg883pkxkhc-en

CONCEJO MUNICIPAL DE SOGAMOSO (2012). Plan de Desarrollo 'Sogamoso Ciudad Competitiva' 2012-2015. Recuperado de http://sogamoso.org/PDM-SOGAMOSO-2012-2015/11\%2O FINAL.pdf

GARCÍA, E. (1998). Infancia, ley y democracia: una cuestión de justicia. En E. García y M. Beloff (comps.), Infancia, ley y democracia en América Latina (pp.3-31). Buenos Aires: Editorial Temis - Depalma.

HERNÁNDEZ, A. (2013). Incidencia de la rendición de cuentas en la gobernanza y la gestión del agua. Un análisis regional de cuatro casos de estudio. Recuperado de http://rendircuentas.org/wp-content/uploads/2013/11/RDCAgua_final-2O-Sep-2013.pdf

HUFTY, M. (2011a). Governance: exploring four approaches and their relevance to research. Research for Sustainable Development: Foundations, Experiences, and Perspectives, pp. 165-183, U. Wiesmann, H. Hurni (Eds.), Geographica Bernensia.

. (2011b). Investigating policy processes: the governance analytical framework. Research for Sustainable Development: Foundations, Experiences, and Perspectives, pp. 403-424. U. Wiesmann, H. Hurni (Eds.), Geographica Bernensia.

JOLLY, J. (2002). Lo público y lo local: gobernancia y políticas públicas. Seminario internacional sobre el fenómeno administrativo público. Bogotá: ESAP.

KOOIMAN, J. (1993). Modern Governance: New Government-Society Interactions. London: SAGE Publications. . (2004). Gobernar en gobernanza. Revista Instituciones y Desarrollo, 16, 171-194.

LE GALÈS, P. (1998). Régulation, gouvernance et territoire. En J. Commaille y B. Jobert (eds.), La régulation politique à paraître (pp. 203-240). Paris: Presses de Sciences Po. 
MADRUEÑO-AGUILAR, R. (2017). Gobernanza, desarrollo y seguridad: trilemas e implicaciones para la coherencia de políticas. Iberoamerican Journal of Development Studies, 6(1), 76-101

MAHONEY, J. (2000). Path Dependence in Historical Sociology. Theory and Society, 29(4), 507-548.

MAYNTZ, R. (2005). Nuevos desafíos de la Teoría de la Gobernanza. En A. Cerrillo (ed.), Gobernanza hoy: 10 textos de referencia (pp. 83-99). Madrid: Instituto Nacional de Administración Pública.

NORTH, D. (1993). Instituciones, cambio institucionaly desempeño económico. México: FCE.

PINEDA, J. (2014). Gobernanza, desarrollo local y calidad del trabajo. Los casos de Cartagena, Pereira y Pasto, 2001-2008. Revista Iberoamericana de Estudios Municipales, 9, 37-72. . (2018). Análisis de políticas públicas en Colombia. Enfoques y estudios de caso. Bogotá: Ediciones Uniandes.

PNUD (1997). Reconceptualising Governance: Discussion paper 2. New York: Management Development and Governance Division, Bureau for Policy and Programme Support, United Nations Development Programme.

. (2014). Discussion paper: Governance for Sustainable Development. Integrating Governance in the Post-2015 Development Framework. New York: United Nations Development Programme. Recuperado de https://www.undp.org/content/dam/undp/library/Democratic\%2oGovernance/Discussion-Paper--Governance-for-Sustainable-Development.pdf

PORTES, A. (2006). Institutions and Development: A Conceptual Reanalysis. Population and development review, 32(2), 233-262.

PRATS, J. (2003). El concepto y el análisis de la gobernabilidad. Revista Instituciones y Desarrollo, 14-15, 239-269.

. (2005). De la burocracia al management, del management a la gobernanza. Las transformaciones de las Administraciones Públicas de nuestro tiempo. Madrid: Instituto Nacional de Administración Pública.

ROTH, A. (2003). Políticas Públicas. Formulación, implementación y evaluación. Bogotá: Ediciones Aurora.

RHODES, R. (1996). The New Governance: Governing without Government. Political Studies, $44(4), 652-667$.

RUANO, J. (octubre, 2002). La gobernanza como forma de acción pública y como concepto analítico. Trabajo presentado en VII Congreso Internacional del CLAD sobre la Reforma del Estado y de la Administración Pública, Lisboa, Portugal.

SABATIER, P. (1999). Theories of the Policy Process. Boulder, CO: Westview Press. 